\title{
Index
}

7 vírgenes 101, 102, 103, 105n4

8 Femmes/8 Women 227, 256

20 centimetros/20 Centimetres 241, 250-3

800 balas $/ 800$ Bullets 54, 60, $63 \mathrm{n} 23,63 \mathrm{n} 24$

399375

A la ciutat/In the City 200

A los que aman/To Those Who Love 224

A tres bandas 31

The Abandoned 41

Abre los ojos/Open Your Eyes 48, 50, 51-2, 53, 55, 62n10, 62n11, 69, 202, 210, 213, 214

Abril, Victoria 3

Acción mutante/Mutant Action 54, 90, 91, 119

Acevedo-Muñoz, Ernesto 160, $175 \mathrm{n} 5,217 \mathrm{n} 9$

Acland, Charles R. 39

action film 4, 29, 53, 54, 90, 127, 129-30, 131n4, 136

Actrius/Actresses 186

Adorno, Theodor 122

L'Age d'Or 204

Agudo, Mariano 95

Agular, Santiago 107, 120n4

see also La cuadrilla

Airbag 91, 135, 136-42, 143, 144, 148-9, 150n4

Akerman, Chantal 184, 186
Al salir de clase 69

Alarcón, Tonio L. 73

Alas de mariposa/Butterfly Wings 137

Alicia 72

Allinson, Mark 2, 44, 49, 138-9, $150 \mathrm{n} 5,175 \mathrm{n} 4$

Almodóvar, Agustín 54, 226

Almodóvar, Pedro xv, xvii-xviii, 2, 9, 10, 11, 17, 44-8, 49, 51, $52,54,56,57,58,59,60,61$, 61n6, 61n8, 63n22, 111, 114, 139, 157-78, 179, 209, 210, 213, 216n2, 226, 228, 235n9, 253, 254, 257n4, 275n5

Altman, Rick 3, 4, 6, 13, 90, 113, 114, 206, 215-16, 243-4, 245, 248, 249, 250, 252

Altman, Robert 184, 235n7

Álvarez, Enrique 288

Álvarez, Miguel 103

Álvarez Monzoncillo, José María 128

Alves, Joe 203

Los amantes del Círculo Polar/The Lovers of the Arctic Circle 194, 220

Amenábar, Alejandro xv, xvii, 2, $12,17,27,44,46,47-8$, 50-2, 54, 55, 56, 60, 61, 69, $70,90,91,138,150$ n 8,179 , $202,205,210,211,213,214$, 215, 216n1, 217n10, 220, 223

The Amityville Horror 74 
Amodeo, Santiago (Santi) 93, 95, 96, 97, 101, 102, 105n4

Amores perros 169

Amo-Sánchez, Antonia 245

Andersen, Bibí 172, 177n17, 253

Anderson, Benedict 181

Anderson, Brad 37

Año Mariano 66

apertura period 8, 110, 118, 180

Appadurai, Arjun 14, 39

Arachnid 32, 33, 37, 40, 81n8

Aranda, Vicente 93, 112, 125, 213, 253

Aranoa, Fernando León de 260

Arau, Alfonso 76

Argento, Dario 74

Aristarain, Alfonso xv

Armendáriz, Montxo 128, 138, 260, 262, 264

Armero, Fernández 67, 90

Armiñán, Jaime de 210, 253

El arreglo 124

Arribas, Marta 260

El arte de morir/The Art of Dying 65, 66-71, 72, 77, 80, 82n12, 199

Assayas, Olivier 49

Astronautas 101, 102, 104, 105n4, 106n 8

¡Átame!/Tie Me Up, Tie Me Down $44,56,57,61 \mathrm{n} 2$

Atilano, presidente $120 \mathrm{n} 4$

Atkinson, Michael 80, 82n12

L'Auberge espagnole/The Spanish Apartment 96

Augustín, Álvaro 77, 81n10

Aunque estés lejos/So Far Away 287

Aurum Producciones 67, 70

Las aventuras de Pepe Carvalho 124

¡Ay, Carmela! 241, 244, 249-50

Aznar, José María 17, 128

Backwoods 42

Bajo Ulloa, Juanma 90, 91, 135, $136,137,138,139$

Bakhtin, Mikhail 10, 11, 188, $201 n 2$
Balagueró, Jaume 41, 65, 66, 71, $72,73,74,75,80,81 \mathrm{n} 5$, $82 \mathrm{n} 12$

Balletbò-Coll, Marta 220

Banderas, Antonio 3, 166

Baquero, Ivana 77, 208

Barbadillo, Pedro 98

Barcelona Olympics 3, 108, 125, 161

Bardem, Javier xvii, 3, 50, 52, $62 \mathrm{n} 19,63 \mathrm{n} 24,137,141$

Bardem, Juan Antonio 2

Barranco, María 157

Begines, Álvaro 97, 100, 105n4, 242

Belbel, Sergi 185, 186, 194, 196

Belinchón, Gregorio 68, 69, 70

Bellmunt, Francesc 186

Beltenebros/Prince of Shadows 126

Beneath Still Waters 27, 41

Benigni, Roberto 76

Benjamin, Walter 244, 245

Bergfelder, Tim 259, 261

Bergman, Ingmar 10, 222, 223

Berlanga, Luis García 2, 6, 18n3, 108, 109, 151n10, 247

Berney, Bob 78, 79

Bernstein, Elmer 228

Bertolucci, Bernardo 123

Besas, Peter 2, 35, 120n2

Beyond Re-Animator 32, 33, 37, 40

Bielinsky, Fabián 91

Bienvenido Mister Marshall $151 \mathrm{n} 10,247$

Bigas Luna 114, 118, 124, 179

biopic 51, 88

Blade 275

Blanks, Jamie 67

blockbuster 56, 112, 135, 136, 137, 139, 141, 148

Bodegas, Roberto 125, 149n2

El bola/Pellet 103

Bollaín, Iciar 179, 260, 265, 266, 268, 269, 272, 276n13

Boquerini 70

Borau, José Luis 9, 204, 211, $275 \mathrm{n} 6$

Borden, Lizzie 57 
Bosé, Miguel 162, 163, 166, 167, 169

El bosque del lobo 120n6

Botting, Jo 70

Boudier, Christian 40

Boyero, Carlos 78

Boyle, Danny 103

Bradshaw, Peter 235n9

Bratton, Jacky 159

Bren Entertainment 31, 36

Brieva, Miguel 97, 98, 102, 106n8

Bronston, Samuel 34-5

Brooks, Peter 159, 228

Brujas 67

Buckley, Christine 50

buddy film 135

Buena Vista 72

Buena Vista Social Club 284

Buñuel, Luis xv, 2, 184, 186, 204, 205, 211, 213

Buse, Peter 2, 62n16, 106n9

Butler, Judith 270

Bwana 260, 276n14

Cabeza de perro/Doghead 97, $105 \mathrm{n} 4,106 \mathrm{n} 8$

Caiga quien caiga 60

Calamaro, Andrés 100

Caldwell, Christopher 275n10

El calentito 103

Calhoun, John 80

Calleja, Pedro 70, 74, 75

Calor . . y celos 290

Cambio de sexo 253, 254

El caminante 95

Camino, Jaime 200

Campano, Paco 105n4

Camporesi, Valeria 221, 234, 246

Caña, Félix 105n4

Cannes Film Festival 71, 78, 79, $235 n 7,274 n 3$

Cantero, Mercedes 93

Cantero-Exojo, Mónica 275n7

Cantó, Toni 172

Caparrós Lera, J. M. 19n6, 20n12

Carabante, Chiqui 93

Carícies/Caresses 181, 182, 187-8, 194-9, 200
Carlos contra el mundo 93

Carmen 93

Carmen, la triana 242, 247-8

Carne trémula/Live Flesh xvii, 57, 161, 174, 176n13

Carrasco, Diego 98

Carroll, Noël 229, 234n5

Carta Blanca 222, 234n4

Las cartas de Alou/Letters from Alou 260, 262-6, 267, 272, 275n6, 276n14

Carvajal, Pedro 290

Casanova, Jorge 94

El caso Almená 125

Il caso Mattei/The Mattei Affair 123

Cassavetes, John 135, 144, 222

Castañeda, Rigoberto 42

Castelao Productions 31, 37

Català, Víctor 88

Catalán, Álex 94, 95

Cavell, Stanley 109

La caza/The Hunt 208, 210

El cerco del diablo/Besieging the Devil 186

Cerdà, Nacho 41

Cervera, Mónica 253

Cettl, Robert 120n7

Chamartín Studios 35

Chaplin, Geraldine 207

Chávarri, Jaime 186, 242

Chavarrías, Antonio 276n14

Choose Me 226

Chungking Express 223, 234n4

El Cid 35

Cinexín 94, 95, 96, 97, 105n5, 106n7

Cobeaga Eguillar, Borja 70

El cochecito 109, 110, 113, 115, 116

Coixet, Isabel xv, 2, 17, 219-38

Coletti, Enrico 31

Collins, Jim 102

Colomo, Fernando 9, 280, 291

comedy 4, 8, 9, 13-14, 53-4, 56, $59,60,61 \mathrm{n} 6,67,89,90,95$, 100-1, 103, 140, 159, 185, 201n2, 247, 261, 289, 291

dark/black 107-21, 136

of errors 137 
comedy (cont.)

Golden Age 137, 140

of manners 135

romantic 133, 137, 280, 287

screwball 48,137

situation (sitcom) 97

slapstick 45, 105n5

social satire 136

workplace 194

see also esperpento

comic books 9, 79, 81, 98, 106n8, 220

coming-of-age film 135, 137

Como agua para chocolate/Like

Water for Chocolate 76

Compañía Industrial Film Español S.A. (CIFESA) 6

Company, Juan Manuel 2, 5, 6-7

Companys, procés a Catalunya 88

Compás, Mártires del 93

La comunidad/Common Wealth 54, 59, 66

Comunidades Autónomas 87, 88, 89,91

Il conformista/The Conformist 123

Coombs, Jeffrey 33

Coronado, José 130

Corrigan, Timothy 73, 222

La corte de Faraón 242

Las cosas del querer/The Things of Love 242

Cosas que dejé en La

Habana/Things I Left in

Havana 260, 267, 280, 285, 292-3

Cosas que nunca te dije/Things I

Never Told You 219, 221, 224, 225-6, 229, 230-1, 233, 234n 3, 235n8

Costa Brava: A Family Album 220

Costa Gavras 123

costumbrismo ibérico 10, 12, 19n10

Courtois, Miguel 129

Coyote, Peter 158

Craven, Wes 66, 67, 70

Cremata Malberti, Juan Carlos 285

Cría cuervos/Cria! 78, 204, 207, 209, 210, 213, 214
Crimen ferpecto/Ferpect Crime 54

La cripta 124

Cronenberg, David 11, 74

Crónicas de las guerras carlistas 88

Cronos 75

Crowe, Cameron 50

Cruise/Wagner Productions 34, 50, 211

Cruz, Penélope xviii, 2, 44, 50, 52, $56,58,59,62 \mathrm{n} 20,158,166$, 174, 210, 248

cruzada 6

La cuadrilla 107, 112, 120n4 see also Luis Guridi; Santiago Aguillar

Cuarón, Alfonso 76, 77

Cuarteto de La Habana/Havana Quartet 280, 291

Las cuatro verdades/The Four Truths 186

Cuentos para una escapada/Stories of an Escapade 186

Cuerda, José Luis 50

Cunill, Inma R. 95

Curro Jiménez 63

Curtiz, Michael 166, 227

Dagon 36-7, 81n8

Dapena, Gerard 179, 188, 199

Darkness 41, 65, 71-5, 77, 80, 81n6, 81n 8

De Alberi, Attilio L. 289

Dechent, Antonio 99

Los Delinqüentes 93

Demasiado caliente para ti 290

Demasiado viejo para morir joven/Too Old to Die Young 219

Deprisa, deprisa 103

Derleth, August 74

Los desafios/The Challenges 186

destape 9, 59

El detective y la muerte 126

Deuce 95

El día de la bestia/The Day of the

Beast 52-3, 54, 60, 62n15, 90, 103, 119, 138

El día que nací yo 242 
Dias contados/Running Out of Time 19n11

Dias sin luz 72

Díaz López, Marina 251

Díaz Torres, Daniel 280, 288, 289

Díaz Yanes, Agustín 138

Díez Clivillé, Joan M. 196

Dimension Films 55, 71-2

Dirty Pretty Things 259, 268, $274 n 1$

Di Salvo, Thomas 267

D'Lugo, Marvin 2, 111, 175n4, 179,291

Do the Right Thing 271

Doane, Mary Ann 164, 175n8, 232, 235n10, 236n18

documentary 94, 95, 97-9, 127, 131, 138, 180, 200, 273, 276n11, 284

Dogma 95 135, 144-5

Domínguez, Juanjo 95

Los dos lados de la cama 251 drama 51, 90, 189, 226, 235n9, 240

corporate 149

marital 194

prison 122

romantic $235 \mathrm{n} 11$

Drove, Antonio 124

Ducastel, Olivier 256

Dyer, Richard 29, 255

Dziga Vertov 183

Ealham, Chris 173

Ebert, Roger 235n9

Egea, Juan 110

Die Ehe der Maria Braun/The Marriage of Maria Braun 205

Eleftheriotis, Dimitris 33

Elejalde, Karra 6, 138

El Fary 14

El Gran Wyoming 14, 60

Elisa, vida mía/Elisa, My Love 210

Elorrieta, Javier 290

Elsaesser, Thomas 48, 49-50, 53, $104,160,175 \mathrm{n} 3$

En la puta calle/Hitting Bottom 260
Entre tinieblas/Dark Habits 157, 213

episode film 179-201

Equipo 'Cartelera Turia' 5, 7, 18n1

Erice, Víctor xv, 78, 100, 138, 204, 206, 207, 209, 213, 214, 216n7

Escamilla, Barbara 69

Escobar, Manolo 19n5

Escrivá, Angeles 276n12

españolada 166, 241, 242, 246, 247-50, 255, 256

esperpento 11, 12, 13, 99, 106n9, 136

El espinazo del diablo/The Devil's Backbone 75, 76, 78, 81n11, 205, 207-8, 209, 214, 216n4

El espiritu de la colmenalThe Spirit of the Beehive xv, 78, 100, 204, 206, 207, 210, 211, 213, 214, 215, 216n7

Esquirol, Meritxell 13

Estudios Picasso 77

ETA $125,129,131,131 n 1$

Etherington, Daniel 235n9

European Union 3, 89, 117, 260, 265, 266, 275n10

Evans, Peter William 2, 257n2

Extranjeras 260, 273-4

El extraño viaje 109, 115

Faces 135

El factor Pilgrim 93, 95, 96, 97, 102

Fantastic Factory 27-43, 71, 72, 74, 75

fantasy $11,20 \mathrm{n} 14,77,79,185$, 190, 194, 205

Le Fantôme de la libertélThe Phantom of Liberty 184, 186

Fassbinder, Rainer Werner 205, 227

Faulkner, Sally 2, 30

Faust: Love of the Damned 32, 33, $38,40,81 \mathrm{n} 8$

Fecé, Josep Lluis 12, 13, 52

Feenstra, Pietsie 245, 247

Félez, José Antonio 95, 105 
Fernández, Fausto 74

Fernàndez, Josep-Anton 180, 181

Fernández, Julio 31, 37, 65, 71

Fernández Armero, Álvaro 65, 66, $67,68,69,70,72,80,81 \mathrm{n} 4$, 90, 199

Ferreri, Marco 108, 109

Filmax 20n14, 27, 31-2, 34, 36, $37,38,41,65,66,71,72$, $81 \mathrm{n} 8,131 \mathrm{n} 4$

film noir 9, 45, 122, 124, 127, $135,137,139,157-78$

Les Films Alain Sarde 62n10

Els Films de la Rambla 181

Final Destination 67

Fineline Features 55

Flamenco $105 \mathrm{n} 2$

Flesler, Daniela 267

Flockhart, Calista 41, 81n5

La flor de mi secreto/The Flower of My Secret 57, 158, 161

Flores de otro mundo/Flowers from Another World 265, 266-73, 276n13, 280

Flores, Lola 100, 242

folklórico 6, 80

Ford, John 205

Fordham, Joe 80

Forn, José María 88, 200

Fosforito: la voz, el genio, la leyenda 98

Four Rooms 183

Fraga Iribarne, Manuel 118

Frágiles/Fragile 41, 73, 74, 81n5

Franco dictatorship xviii, 5, 6, 8, $18,30,35,58,88,91,92,99$, $103,111,118,122,124,125$, 126, 127-8, 129, 158, 160, 173, 174, 177n20, 201n2, 204, 207, 209, 212, 213, 242, 244-5, 247, 249-50

Un franco, 14 pesetas 276

Frankenstein 203, 206

Frears, Stephen 103, 259

Fresnadillo, Juan Carlos 2

Freud, Sigmund 122, 167, 203

La fuga de Segovia 125

Fugitivas/Fugitives 91
Fulano y Mengano 99, 106n10

La furia de Mackenzie 105n4

Furtivos 204, 209, 211

Future Films 37

Gabriel, Enrique 260

GAL: en el punto de mira 129, $130,131,131 \mathrm{n} 5$

Galloway, Stephen 76, 78, 79

gangster film 29, 103, 122, 133, 135

Gaos, Lola 211

Garci, José Luis 9, 66, 128, 149n2

Garcia, Andy 93

García, José 130

García, Rodrigo 185

García Bernal, Gael 58, 162-3, 168,169

García Canclini, Néstor 279, 282

García Lorca, Federico, 93, 105n2, 211

García Sánchez, José Luis 242

Gardela, Isabel 276n14

Garrido, Álvaro 94

Gatliff, Tony 93

Gay, Cesc 200

Gehring, Wes 110

Gelder, Ken 96, 97

Genette, Jean 10

Gil, Mateo 101, 213

Gillespie, Jim 67

Gillespie, Marie 279

Gingold, Michael 72

La giralda perdida de Nueva York 98, 99

Girard, François 183, 185

Gitano 93

Gledhill, Christine 4, 62n13, 133, 170, 235n10, 236n15, 261-2, 269

Godard, Jean-Luc 10, 184

Gómez, Fernando Fernán 109

Gómez Pereira, Manuel 112

González, Felipe 125, 131

González Iñárritu, Alejandro 76, 169

Gordon, Paul 204

Gordon, Stuart 22, 81n8 
Goya Awards 76, 212, 219, 257n1

Gracia, Sancho 59, 63n24

Grant, Barry Keith 28

Grant, Catherine 73

Green, Jennifer 65, 66, 71, 81n9

Griffith, D. W. 184, 205

Grivas, Alexis 76

Gual, Roger 135, 143, 144-5, 150n8, 152n14

Guardiet, Romà 88

Gubern, Román 2, 6, 8

Guerin, José Luis 179

Guillén Cuervo, Fernando 66

Guillot, Eduardo 70

Guridi, Luis 107, 120n4 see also La cuadrilla

Guthmann, Edward 235n9

Gutiérrez, Chus 103, 260

Gutiérrez Aragón, Manuel 260, 267, 280, 292

Gutiérrez Caba, Emilio 59

Habana Blues 280, 281, 293-5

Hable con ella/Talk To Her 50, 58, 71,157

Hacerse el sueco 280, 288-90

Hall, Stuart 270, 284

Hartley, Hal 225, 226, 231

Harvey, David 147

Harvey, Penelope 112

Havanera 1820291

Hayward, Susan 14, 15

Hellboy 75, 76

Heredero, Carlos F. 2, 220

Hermida, Tania 105n4

Hermoso, Miguel 91, 93

Herrera, Lola 255

Herzog, Werner 223

Higginbotham, Virginia 2

High Fidelity 103

Higson, Andrew 14, 16, 212 , $217 \mathrm{n} 8$

Hillier, Jim 229

histórico-imperial 6

Hitchcock, Alfred 30, 164, 183, 193, 202, 204, 216n5

Hjort, Mette 144-5

Hoefert de Turégano, Teresa 282
Holland, Jonathan 66, 70, 74

Holmlund, Chris 225, 229

Hopewell, John 2

horror xiv, xv, 4, 6, 7, 9, 11, 17, 20n14, 27-43, 50-1, 52-3, 55, 62n12, 65-83, 89, 91, 107-21, 133, 186, 202-18

Hou, Hsiao-Hsien 49

Huerga, Manuel 104, 128, 179

Huevos de oro 119

Husbands 135

Hutchings, Peter 29, 67, 68, 81n2

\section{Know What You Did Last} Summer 67, 70

Iglesia, Álex de la 44, 46, 48, 52-4, 59, 60, 61, 61n 7, 61n 8,65 , 90, 99, 106n9, 119, 138, 179, 220, 223

Iglesias, Alberto 49

Iglesias, Carlos 276

Iglesias, Gervasio 93, 97, 105

Imagine Entertainment 38

Imitation of Life 232

immigration film 17, 102, 259-78, 292

In the Soup 226

independent (indie) film 17, 56, 107, 111, 135, 144-5, 180, 225-6, 228-30, 234, 235n7, 235n9, 235n12

Instituto Cubano de Arte e Industria Cinematográficos (ICAIC) 282, 286

internet 15, 47-8, 49, 77, 101, 221-2

Jäckel, Anne 40

Jameson, Fredric 10, 14

Jamón, jamón 114, 119

Jarmusch, Jim 185, 222, 225, 226, 231

Jeanette 100

Jeanne et le garçon formidable 256

Jencks, Charles 150n5

Jiménez, Lucía 69

Jones, Alan 80

Jones, Doug 209 
Jordan, Barry 2, 3, 242, 247

Joselito 19n5

Jousse, Thierry 223

Joves/Youth 200

Jules et Jim 233

Jurado, Rocío 242

Justino, un asesino de la tercera edad 107-21

Kassovitz, Matthieu 91

Kawin, Bruce 203, 211, 214

Kazan, Elia 158, 175n2

Keathley, Christian 223-4

Kermode, Mark 80

Las Ketchup 100

Kiarostami, Abbas 49

Kidman, Nicole 34, 50, 52, 53, 202, 211, 212

Kika 158, 210

Kilómetro 3142

Kinder, Marsha 2, 120n1, 120n2, $150 \mathrm{n} 5,166,167,168,175 \mathrm{n} 4$

Klapisch, Cédric 96

Klein, Andy 183

Kleinhans, Chuck 229

The Kovak Box 41

Kristeva, Julia 10

Kubrick, Stanley 74

Labanyi, Jo 2, 30

El laberinto del fauno/Pan's

Labyrinth xv, 65, 75-80,

81n11, 81n12, 100, 205, 208, 209, 211, 214

Laberinto de pasiones/Labyrinth of Passions 157, 174

Lacan, Jacques 167

Laclau, Ernesto 270

Lane, Christina 235n12

Langford, Barry, 133, 134

El lápiz del carpintero 128

Lardín, Rubén 74, 75

Lavadora 102

Lazaga, Pedro 150n6

Lázaro-Reboll, Antonio 2, 7

Leblanc, Tony 13

Lee, Ang 76

Lefebvre, Henri 146
Lena 91

Letter from an Unknown Woman 169, 232, 236n19

Lévi-Strauss, Claude 247

La ley de la memoria histórica 127

La ley del deseo/Law of Desire 44, 158, 176n13, 176n14, 213, 252

La ley de Extranjería 260, 274n2

La ley Miró (Miró Law) 3, 9, 90, 119n1

Linares, Carmen 255

Lions Gate Films 38, 55

Lista de espera/Waiting List 287

literary adaptation 88, 100, 124, 158, 186, 187, 196, 248

Lluvia en los zapatos/If Only 220

Lo, Ismael 49

El Lobo 129, 130, 131n4

Lola, la película 93

Loma, José Antonio de la 103

López, Ana M. 145

López, Sergi 3, 208, 259

Lucia, Luis 242, 254

La Lupe 49

El Lute 125

El Lute II 125

La luz prodigiosa 93

Lynch, David 11

The Machinist 37

McKellar, Don 183

MacNab, Geoffrey 65

Madonna 254, 255

La madre muertalThe Dead Mother 90, 137

Madregilda 126

Madrid, Luis de la 27, 38

magical realism 190

Maite 292

La mala educación/Bad Education 49-50, 58, 61n6, 159, 161-5, $166,168-75,175 \mathrm{n} 9,176 \mathrm{n} 14$, $177 \mathrm{n} 18,213$

Malberti Cabrera, Iraida 285

Mambi 292

Mañas, Achero 103

Mankiewicz, Joseph L. 186 
Mar adentro/The Sea Inside xvii, 50-1, 55, 62n19, 216n1

Marchant, Joaquín Romero 99

Marindas asesinas 54

Marini, Alberto 37, 38

Marisol 19n5, 242, 254, 255, 257n2

Marks, Laura 279

Marsh, Steven 2, 30, 201n2

Martín (Hache) xv

Martin, Michael T. 288

Martín-Cabrera, Luis 272, 273

Martineau, Jacques 256

Martínez, Fele 69, 81n5

Martínez Lázaro, Emilio 71, 186, 241

Marton, Andrew 35

Mata Moncho, Juan de 2, 5, 19n5

Matador 44, 114, 120n6, 177n17, 210,213

Matar al Nani 125

Matías, juez de línea 120n4

Maule, Rosanna 50

Maura, Carmen 59, 158, 172, 249, 253

Maurice, Thierry 245

Medem, Julio 138, 139, 194, 220

melodrama 4, 16, 45, 56, 100 , $101,128,131 \mathrm{n} 1,135,157-$ 60, 163, 165-6, 168, 169, 174, 175, 175n 3, 180, 185, 190, 204, 219-38, 260, 2612, 263, 266, 267, 269-70, 272, 274

Bollywood xiv

indie 221

Méndez Casanova, Antonio 136

Mendik, Xavier 32, 34

Mendoza, Eduard 124

Mercer, John 236n15

El método/The Method 149n2

Metropolis 205

Metz, Christian 51

Mi casa es tu casa 103

Mi querida señorita 253

Mi vida sin milMy Life Without

Me 219, 221, 222, 223, 2245, 226-33, 234n3, 235n9, $235 \mathrm{n} 11$
Miel para Oshún/Honey for Oshun 285, 291

Mildred Pierce 166, 168, 227

Miller, Michael 235n9

Miller, Toby 279

Mimic 75

Miradas 288

Miralles, Melchor 129, 130, 131n4

Miramax 44, 56, 57, 63n21, 63n22, 71, 229

Miret, Rafael 235n8

Miró, Pilar 126, 213

misionero 6

Mitchell, Robert 76

Modleski, Tania 204

Molina, Manuel 97

Molina-Foix, Vicente 2

Molina Gavilán, Yolanda 267

Mollà, Jordi 101, 130

Monterde, José Enrique 10-11, 20n12, 103

Monzó, Quim 185, 186

Monzón, Daniel 41

Moreiras Menor, Christina 53

Moreno, José Luís 14

Morgan-Tamosunas, Rikki 2, 3 , 242, 247

Morir (o no)/To Die (Or Not) 181, 182, 194, 199, 200, 201n3

Morricone, Ennio 49

Morris, Barbara 2

la movida 161, 162, 245, 251

Muerte en Granada/The Disappearance of Federico García Lorca 93

Muertos de risalDying of Laughter 54, 59

Mujeres al borde de un ataque de nervios/Women on the Verge of a Nervous Breakdown 44, 56, 61n6, 157

Mulligan, Robert 215, 217n10

music 49, 55, 58, 93, 96, 97, 101-5, 105n3, 113-14, 140, 141, 143, 148, 166, 196-7, 216n1, 228, 241, 243-4, 246-7, 248, 250, $256,257 \mathrm{n} 5,287,289,293$, 294, 295 
music videos 59, 94, 220, 244

musical 4, 6, 17, 19n5, 31, 100-1, $133,140,159,187,241-58$

Musté, Pedro Costa 125

Nada en la nevera 67

Nadie conoce a nadie/Nobody Knows Anybody 101, 214

Nadie hablará de nosotros cuando hayamos muerto 138

Naficy, Hamid 220, 279

Navajas, Gonzalo 274n1

Navarro, Antonio José 73, 74

Navarro, Bertha 76

Nazzaro, Joe 80

Neale, Steve 4, 13, 46, 227, 231

New Line Cinema 67, 183

Nichols, Bill 134, 135, 138

El nido/The Nest 210

Nimri, Najwa 56

La niña de tus ojos/The Girl of Your Dreams 241, 244, 247-9

NO-DO (Noticiario Documental) xviii, 97, 248

No me pises que llevo chanclas 93, 100

Nora, Pierre 244

Noriega, Eduardo 56, 101, 130, 210

Los nuevos españoles 149n2

The Nun 27, 38-9, 41

Objectivo 6735

Ocaña, retrat intermitent 180-1, 200n1

La ola 288

Olasagasti, Eneko 292

Olea, Pedro 120n6, 242

On connaît la chanson/Same Old Song 255, 256

Operación Ogro 125

L'Orgia 88

Orion 44

Ortiz, Florencio 94

Ortuño, José Francisco 98

The Other $217 \mathrm{n} 10$

El otro lado de la cama/The Other

Side of the Bed 71, 241, 250, 251-3, 255
Los otros/The Others xv, 27, 34, $50,53,62 n 11,62 n 12,202-$ 18,220

Ozon, François 227, 256

Paddington, Bruce 288

Palacios, Jesús 11-12, 20n14

Palacios, Manuel 93

Palma, Rossy de 253, 254

Paredes, Marissa 158

Pareja de tres 31

Paris, je t'aime 182, 233

Paris vu par . . 182, 196

Partido Popular (PP) 3, 17, 128, $129,130,162,173$

Partido Socialista Obrero Español (PSOE) 3, 18, 108, 119n1, 128, 129, 162, 173

La pasión turca 112

Pastel de sangre/Blood Cake 198

Pastori, Niña 100

Pata Negra 93

Pataky, Elsa 37, 69

Pávez, Terele 59

Películas para no dormir 41, 42

Peña, Candela 158

Pepi, Luci, Bom y otras chicas del montón 174

Perdita Durango 54, 60, 63n24, 220

Pérez, Ana 260

Pérez, Fernando 280, 285, 286, 287

Peris-Mencheta, Sergio 69

Perkins, V. F. 30

Perojo, Benito 242

El perquè de tot plegat/What's It All About 181, 182, 188-94, 195,200

Perren, Alisa 56, 63n21

Perucha, Pérez 235n8

Picturehouse 77, 78, 79

Piña, Begoña 68

Piñeyro, Marcelo 149n2

PiPiPiPiPiPi o el Teorema de la Felicidad 95

El pisito 108, 115

Plácido 108, 113 
Los Planetas 93

Plaza, Paco 37, 38

Polley, Sarah 219, 226, 228, 229, $230,234 n 6,235 n 9$

Poniente 260, 276n14

Pons, Ventura 17, 88, 179-201

Pont de Varsòvia/Warsaw Bridge 199

Pontecorvo, Gillo 125

¿Por qué se frotan las patitas? 97, $100,101,105 \mathrm{n} 4,241-2,250$ 1,255

pornography $18 \mathrm{n} 1,89$ see also destape

Portabella, Pere 179, 199

Poster, Mark 61n3

post-Franco era 9, 88, 92, 111, 119n1, 125, 126, 129, 161, $166,173,179,180,185$, 200n1, 210, 244, 253

Pozuelo, Juan Vicente 66, 67, 68, 70

Prades, Jaime 35

Princesas 260

Las Producciones del Escorpión 34, $50,62 \mathrm{n} 10$

Promotora de Informaciones, S.A. (PRISA) 129

Pyramid 38

¿Qué he hecho yo para merecer esto?/What Have I Done to Deserve This? 158, 257n4

¿Qué tan lejos! 105n4

Qué t’hi jugues Mari Pili? 88

Queen 254

Querejeta, Elías 19n6

Quintana, Ángel 9-10, 11-12, 19n11, 234

Radhakrishnan, R. 271

Ramos, Francisco 66, 67

Ramos, Santiago 130

Raphael 100

Un rayo de luz 242

Real, Cayetano del 124

Rebollo, Javier 290

Recha, Marc 179
Regueiro, Francisco 126

Reina Zanahoria 149n2

Reinoso, José Luis 105n4

Reixa, Antón 128

Resnais, Alain 123, 255

Rey, Florián 242, 248

Riambau, Esteve 2, 9, 18, 120n1, $120 \mathrm{n} 2$

Ribas, Antonio 88

Ricciarelli, Cecilia 288

Rich, R. Ruby 283

Richards, Michael 173

Rio abajo/On the Line 275n6

Ríos, Santiago \& Teodoro 292

Ripoll, María 2, 194, 220

Ritchie, Guy 91, 103

road movie 91, 135, 136, 137, 142,261

Rocha, Glauber 120n3, 205

Rockwell, Alexandre 225, 226

Rodríguez, Alberto 94, 95, 96, 99, $100,101,105 \mathrm{n} 4$

Rodríguez, Mala 93

Rodríguez, María Pilar 273

Rodríguez Marchante, Oti 53

Rodríguez Merchán, Eduardo 248

Rodríguez Ortega, Vicente 50

Rodríguez Zapatero, José Luis 17, $127,177 \mathrm{n} 20$

romance $56,185,187,261$

transnational 279-96

Romasanta: The Werewolf Hunt $37-8,40,41$

La Ronde 184, 186, 194

Rosenberg, Stuart 74

Rossi, Francesco 123

Roth, Cecilia 158, 174

Rottweiler 32

Roxborough, Richard 41

Royo, Francisco Javier 66, 67, 68, $70,81 \mathrm{n} 4$

Rudolph, Alan 225, 226

Ruiz 'Queco', Manuel 100

Russell, Jamie 235n9

Sabor latino 290

Sacristán, José 124

Saïd 260, 276n14 
Sakamoto, Ryuichi 49

Salazar, Ramón 241

Salmerón, Gustavo 68

Salvador (Puig Antich) 104, 128

San Juan, Antonia 159

San Sebastián Film Festival 95

Sánchez, Julio 95

Sands, Julian 37

Santamaría, Enrique 274n2

Santamarina, Antonio 2, 220

Santaolalla, Isabel 2, 268, 269, $275 n 4,292$

Sanz, Jorge 130

Saraband 223

Sardá, Rosa María 195

Saura, Carlos xv, 2, 6, 18n3, 19n6, 78, 93, 103, 105n2, 138, 204, 207, 208, 209-10, 213, 214, 241

Schein, Louisa 279

Schnitzler, Arthur 204

Scorsese, Martin 103

Scott, A. O. 235n9

Scream 66, 67, 68, 69, 70-1, 77

Scream 267

Scream 367

The Searchers 205

Second Name 37

Segura, Santiago 13-14, 60, 150n4

Sr Chinarro 93

serial killer film 107, 108, 110, 114-16, 119, 120n6, 120n7, 177n17, 206

Serra, Elena 37, 38

Serra, Koldo 42

Serrano, Julieta 195

Serrat, Joan Manuel 148

Sesto, Camilo 100

Sevilla World Expo '92 3, 92-3, 104, 105n2, 108, 112, 125, 162

Sevillanas $105 \mathrm{n} 2$

Sex, Lies, and Videotape 56, 57, $235 \mathrm{n} 7$

sexy celtibérica 6

Sharian, John 37

Shingler, Martin 236n15

The Shining 74
Shohat, Ella 14, 279

Sholder, Jack 32, 33, 81n8

Short Cuts 184, 235n7

Silencio roto/Broken Silence 128

Sinfonica española 35

Sin nombre/The Nameless 71

Sirk, Douglas 30, 160, 232

Skinner, Frank 228

Sklar, Robert 61n1

Smash 97

Smith, Adam 134, 149n1

Smith, Kevin 103

Smith, Paul Julian 2, 126, 176n14, $181,189,216 \mathrm{n} 7,217 \mathrm{n} 9$

Smoke 226, 231, 235n7

Smoking Room 133, 142-9, 149n2, 150n8, 151n10

Sociedad General de Cine S.A. $62 \mathrm{n} 10$

Soderbergh, Steven 56, 57, 235n7

Sogecine 34, 129

Soja, Edward 145-6

Solas 91, 100

Solás, Humberto 285, 288

Soldados de plomo 124

Soler, Llorenç 260

Solitud 88

Sony Pictures Classics 44, 45, 58, 59,226

Spanish Civil War 25, 111, 127-8, 131, 205, 206-8, 210, 212, 216n4, 222, 242, 244-5, 24750

Spanish-Cuban co-productions 17, 266-73, 279-96

Stam, Robert 11, 14, 279

Stavisky 123

Stock, Ann Marie 283

Stone, Rob 2, 138

Stranger Than Paradise 226

Suárez, Gonzalo 126, 149n2

Suite Habana 285

Susanna 276n14

Suspiros de España 242

Taberna, Helena 264, 273, 274, $277 \mathrm{n} 19$

Tabío, Juan Carlos 287 
Tacones lejanos/High Heels 44, 158, 161-4, 165-8, 175, $176 \mathrm{n} 13$

Talens, Jenaro 2

Tapia, Gonzalo 91

Tarantino, Quentin 91, 103, 137

Tatuaje 124

Taylor, Lili 225, 228, 229, 231

television $14,15,16,31,41,42$, $46,49,59-60,63 n 24,69,72$, 76, 77, 78, 81, 81n 3, 87-9, 91, 94, 98, 105n $3,124,127$, $128-30,137,177,222-3$, 226,283

Canal+ 67, 106n7, 129, 130, 144

Canal Sur Televisión 92, 97

Corporació Catalana de Radio i Televisió (CCRTV) 88

Euskal Irrati Telebista (EITB) 88

Quatro 129

Radio Televisión de Andalucía (RTVA) 92

Sogecable 129

Telecinco 69, 77, 130

Televisión de Galicia 36

TVE (Televisión Española) 35, $67,94,144,212,282$

TVE2 81n 4,223

TV3 97

Telotte, J. P. 170, 176n10

Tequila 252, 257n5

Térmens, Ramon 200

Tesela Producciones

Cinematográficos 95, 99, 103, 105

Tesis/Thesis 50, 51, 55, 69, 90, 91, 138, 202, 210, 213, 214

La teta $i$ la lluna 119

thriller 9, 13, 17, 42, 48, 50-1, 56, $59,60,70,133,137,159$, 199, 202, 259, 261

political 122-32

thriller policíaco 180

To Kill a Mockingbird 215, $217 \mathrm{n} 10$

Todo es mentira 67, 90

Todo por la pasta 90, 91
Todo sobre mi madrelAll About My Mother 44, 45, 50, 58, 59, $158,160,167,172,174$, $175 \mathrm{n} 1,216 \mathrm{n} 2,254$

Todos los hombres sois iguales 112

Tomándote 276n14

Tómbola 254

Tonel (Antonio Eligio Fernández) 284, 295

Toro, Guillermo del xv, 65, 66, 75, 76, 77, 78, 79, 80, 81n11, 91, 100, 205, 207, 208, 209, 214, 216n 4

Torras, Carles 200

Torreblanco, Frida 77

Torreiro, Casimiro 2, 8

Torrent, Ana 206, 207, 210

Torrente: el brazo tonto de la ley 13, 60, 150n4

Torrente 2: misión en Marbella 13, $150 \mathrm{n} 4$

Torrente 3: el protector 150n4

Tote King 93

Toute une nuit 184

Traição/Betrayal 196

El traje 99, 100, 101, 105n4

El tren de la memoria 260, 276

Triana Toribio, Núria 2, 3, 7, 30, 54, 62n16, 220, 221, 228, 234, 234n $2,236 n 17,246$, $257 \mathrm{n} 2,257 \mathrm{n} 4$

Trueba, Fernando 9, 241, 248, 249

Truffaut, François 222, 223, 233

Trust 226

Tsai, Ming-Liang 49

Tudor, Andrew 28, 67

Tuduri, José María 88

Turek, Ryan 80

El turismo es un gran invento $150 \mathrm{n} 6$

Tzioumakis, Yannis 225

Un chien andalou 204, 205

El underground: la ciudad del arco iris $98,102,106 \mathrm{n} 8$

Urban Legend 67

Urbizu, Enrique 90 
Uribe, Imanol 19n11, 125, 131n1, 138,260

La vaca lechera 95

Valencia Pictures 65

El valle de los caídos 35

Vallés, José María 186

Valverde, Junio 208

Vanaclocha, José 2, 5

Vanilla Sky 50

Vega, Paz 3

Veloso, Caetano 49

Vengo 93

Venice Film Festival 52, 235n7

Verbeke, Natalia 130

La verdad sobre el caso Savolta 124

Verdaguer, Antoni 31, 291

Las verdes praderas $149 \mathrm{n} 2$

El verdugo 109, 113, 115, 118

Vernon, Kathleen M. 2, 175n4

Vertigo 164

Veruga, Vicente 2, 5, 18n3

iVictoria! 88

La vida es silbar/Life is to Whistle 280, 285, 286-7, 291, 294

La vida secreta de las palabras/The Secret Life of Words 219, 222, 223, 229, 231, 234n3, 236n16

Vidal, Belén 231

video games 15

Villar, Carmen 68

Villar-Hernández, Paz 264, 272

Vincendeau, Ginette 29

Vinterberg, Thomas 144

Le Violon rouge/The Red Violin 183

Viridiana 109, 115, 211

La vita è bella/Life is Beautiful 76

Viva Cuba 285

Volver 44, 50, 58, 59, 176n12

von Trier, Lars 135, 144

Wallovits, Julio 135, 143

Wanda Films 286

Wang, Wayne 226, 231, 235n7

war film 36

Warner Bros Pictures 77
Watling, Leonor 3, 231, 233

Wenders, Wim 49, 284

western 4, 7, 28, 33, 36, 63n23, 133,187

spaghetti xiv, $6,7,18 \mathrm{n} 3,29,33$, 60

Whale, James 203, 206

Wild Bunch 77, 79

Wilder, Billy 163, 169, 222, 223

Williams, Christopher 261

Williams, Linda 4, 62n13, 167 , 168, 204, 231, 233, 235n10, 262, 263

Williamson, Kevin 66, 70

Willis, Andrew 2, 7, 62n16

Wittgenstein, Ludwig 108, 109

Wong, James 67

Wong, Kar-Wai 49, 184, 222, 223, 228, 234n 4

Woo, John 137

Woods, Eva 31

Working Girls 57

Wright, Will 28

Xavier, Ismael 205, 212

Y tu mamá también 76

Yang, Edward 49, 184

Yarza, Alejandro 160, 175n5, 216n3, 217n9

Yeh, Yueh-Yu 49

You're the one (una historia de entonces) 66

Ying xiong/Hero 76

Yuzna, Brian 27, 32, 33-4, 41, $81 \mathrm{n} 8$

Z 123

Zabala, Carlos 292

Zambrana, Manuel 74

Zambrano, Benito 91, 100, 280, 293, 294, 295

Zanfoña Producciones, La 87-106

Zayas, Daniel de 95

Zhang, Yimou 76

Zorrilla, J. L. 124

Zunzunegui, Santos 2

Zurinaga, Marcos 93 